\title{
ПРИМЕНЕНИЕ МАТЕМАТИЧЕСКОЙ СТАТИСТИКИ В ОБЛАСТЯХ ПИЩЕВОЙ ПРОМЫШЛЕННОСТИ
}

\section{APPLICATION OF MATHEMATICAL STATISTICS IN THE FOOD INDUSTRY}

\section{Kandalova \\ V. Bey}

Summary. The food industry is one of the most important in the economic structure of the Russian Federation and the significance of its research is due to the country's dependence on food supplies. At the same time, the food industry is characterized by a rather complex technological cycle and has many economic entities involved in the sale of products of this industry. In these conditions, the construction of an optimal management system of a food enterprise cannot be carried out without the presence of formed practical models of analysis that help to identify the patterns of their functioning. At the same time, it is the food industry that seems to be the reference for the use of statistical data in it, which allows us to take into account the laws and established trends in the development of forecasts for the development of this and other industries. Mathematical analysis of statistical data of the food industry is based on various models and methods, among which the advantage is given to economic-mathematical or simulation modeling. Using traditional mathematical models, when conducting static observations, trends and forecasts of the development of the industry are built, the study of which is aimed at this article.

Keywords: food industry, trends, dynamics, growth, mathematical model, trend.

\author{
Кандалова Марина Андреевна \\ Ассистент, Московский государственный \\ университет пищевых производств \\ renemaas@yandex.ru \\ Бею Вероника Владимировна \\ Ассистент, Московский государственный \\ университет пищевых производств
}

Аннотация. Пищевая промышленность является одной из важнейших В экономической структуре Российской Федерации и значимость ее исследования обусловлена зависимостью страны от продовольственных запасов. В то же время пищевая промышленность характеризуется довольно сложным технологическим циклом и имеет множество субъектов экономики, участвующих в реализации продукции данной отрасли. В этих условиях построение оптимальной управленческой системы пищевого предприятия не может производиться без наличия сформированных практических моделей анализа, способствующих выявлению закономерностей их функционирования. В то же время именно пищевая промышленность представляется эталонной для применения в ней статистических данных, которые позволяют учитывать законы и сформировавшиеся тенденции при разработке прогнозов развития этой и других отраслей. Математический анализ статистических данных пищевой промышленности строится на различных моделях и методах, среди которых преимущество отдается экономико-математическому или имитационному моделированию. Посредством традиционных математических моделей при проведении статического наблюдения строятся тренды и прогнозы развития отрасли, на изучение которых нацелена данная статья.

Ключевые слова: пищевая промышленность, тенденции, динамика, рост, математическая модель, тренд.

а также объем промышленного производства. Так на рисунке 1 приведена динамика численности работников в промышленности в целом и пищевой за период 2003-2020 гг.

Рис. 1. показывает, как и в целом по промышленности, в пищевой в 2020 году в вязи с кризисными явлениями произошло снижение численности работников. В целом, численность работающих в отрасли на протяжении 2003-2019 гг. стабильно возрастала, несмотря на снижения в других отраслях в отдельные периоды. Однако 2020 год негативно повлиял на все предприятия промышленности, что сказалось на численности сотрудников, часть которых потеряла работу в виду банкротства компаний либо сокращения. Интерес 


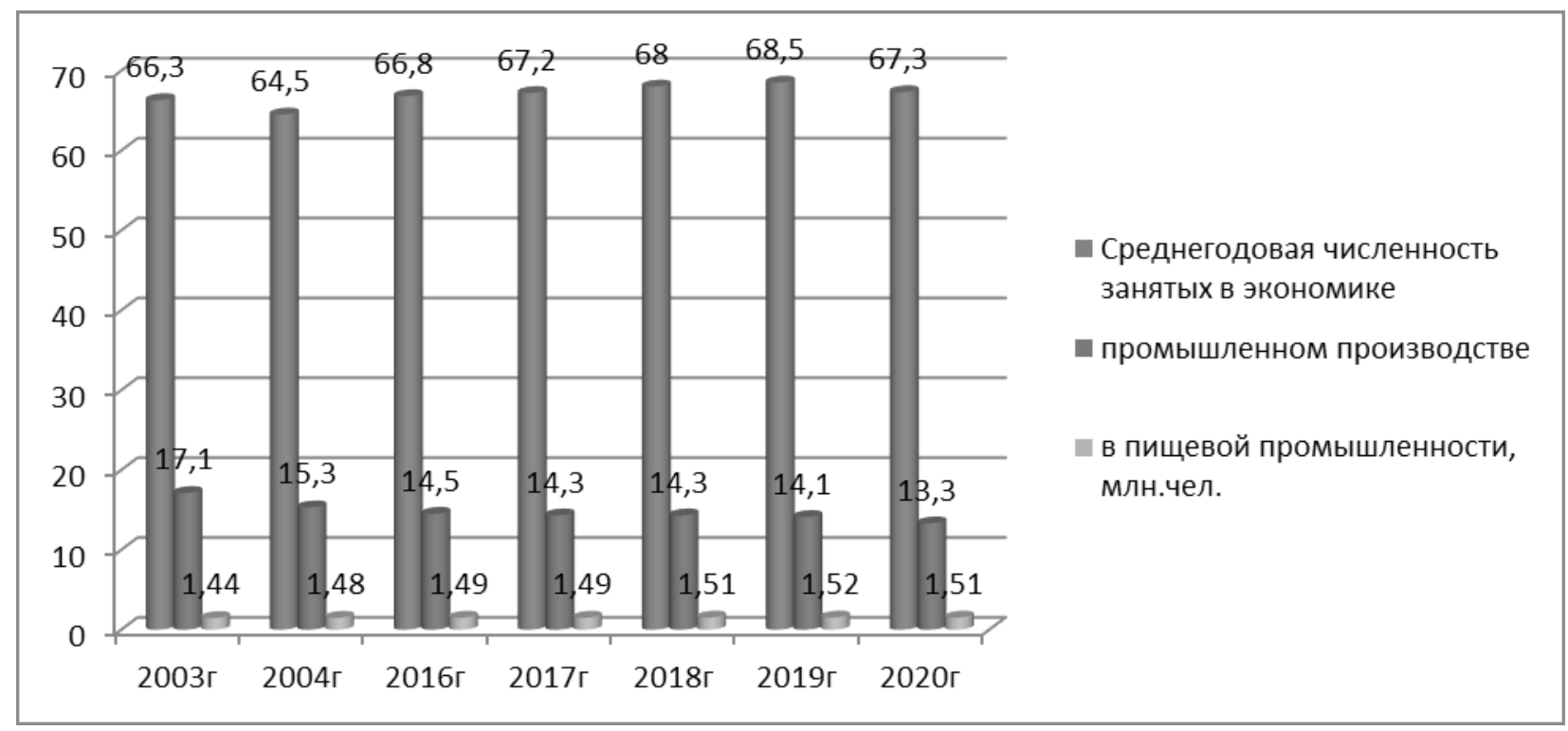

Рис. 1. Динамика численности работников в промышленности в целом и пищевой за период 2003-2020 гг., млн. чел. Источник: составлено автором по [2]

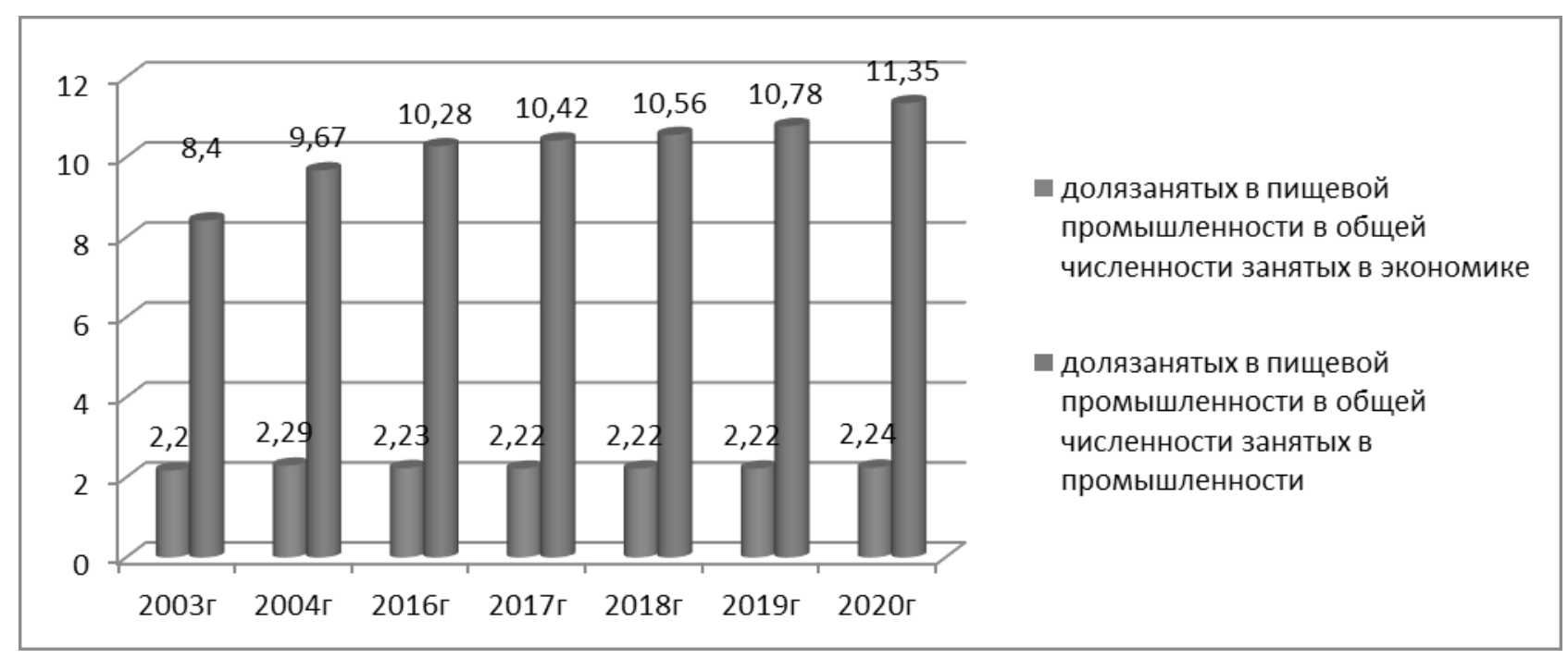

Рис. 2. Соотношение удельных весов в общей численности занятых в пищевой промышленности и промышленности в стране в целом,\% Источник: составлено автором по [2]

представляет показатель удельного веса сотрудников пищевой промышленности в общей численности занятых в экономике и в промышленности целом.

Доля работающих в пищевой промышленности составляет около 10\% от всех занятых в промышленности, что говорит о значимости отрасли. Постепенно наблюдается положительная динамика численности работников в пищевой промышленности, что говорит о расширении пищевой промышленности России. Со- поставление численности по пищевой промышленности, промышленности в целом и всего занятного населения России приведена на рисунке 2.

Доля занятых в пищевой промышленности не значительно увеличилась в отношении всей экономической структуры страны, а в промышленности, произошел довольно ощутимый рост данного показателя, что позволяет сделать вывод о стабильности развития отрасли. Большая часть сокращения сотрудников в 2020 году кос- 


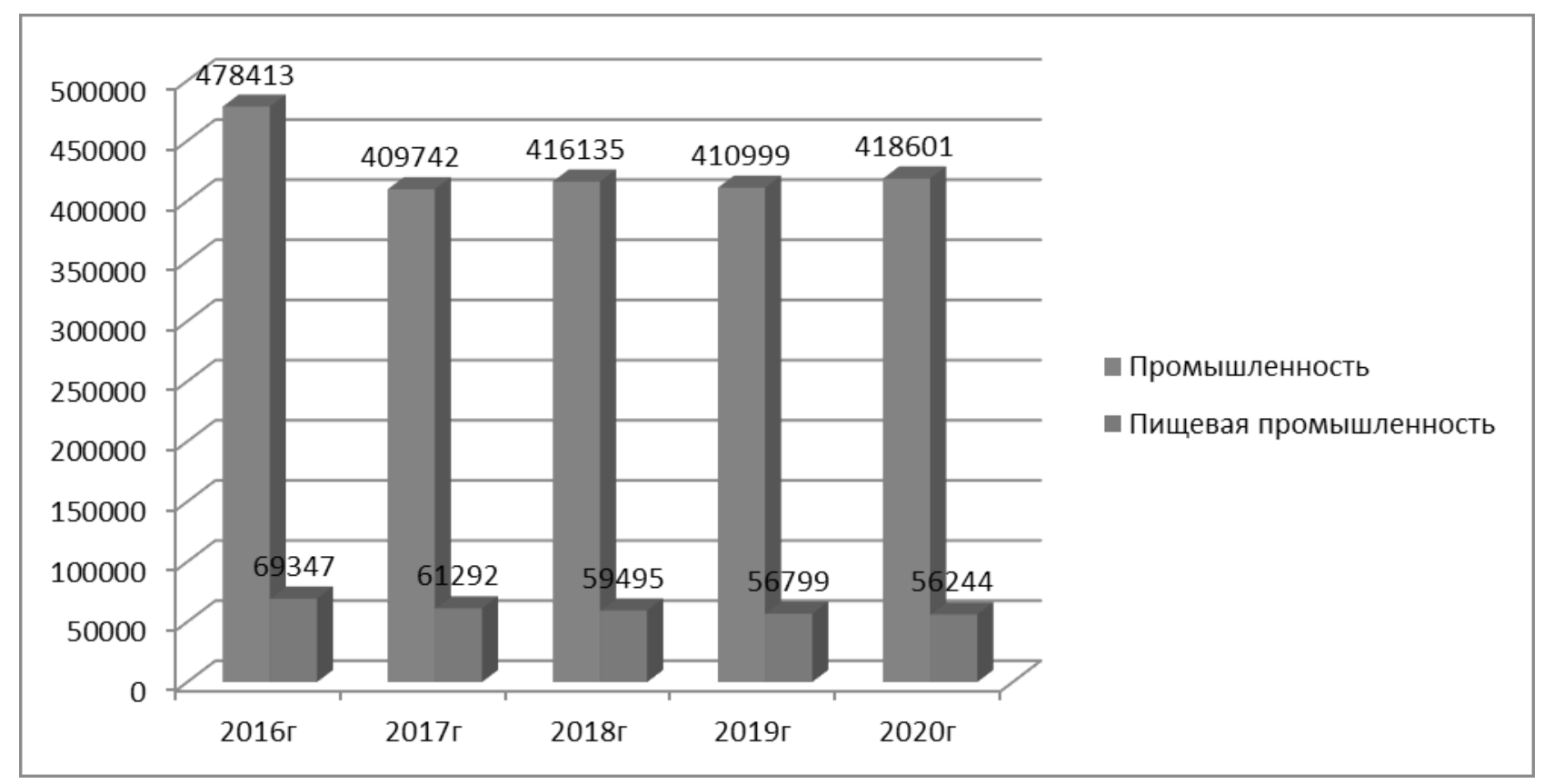

Рис. 3. Динамика числа предприятий в промышленности и пищевой, ед. Источник: составлено автором по [2]

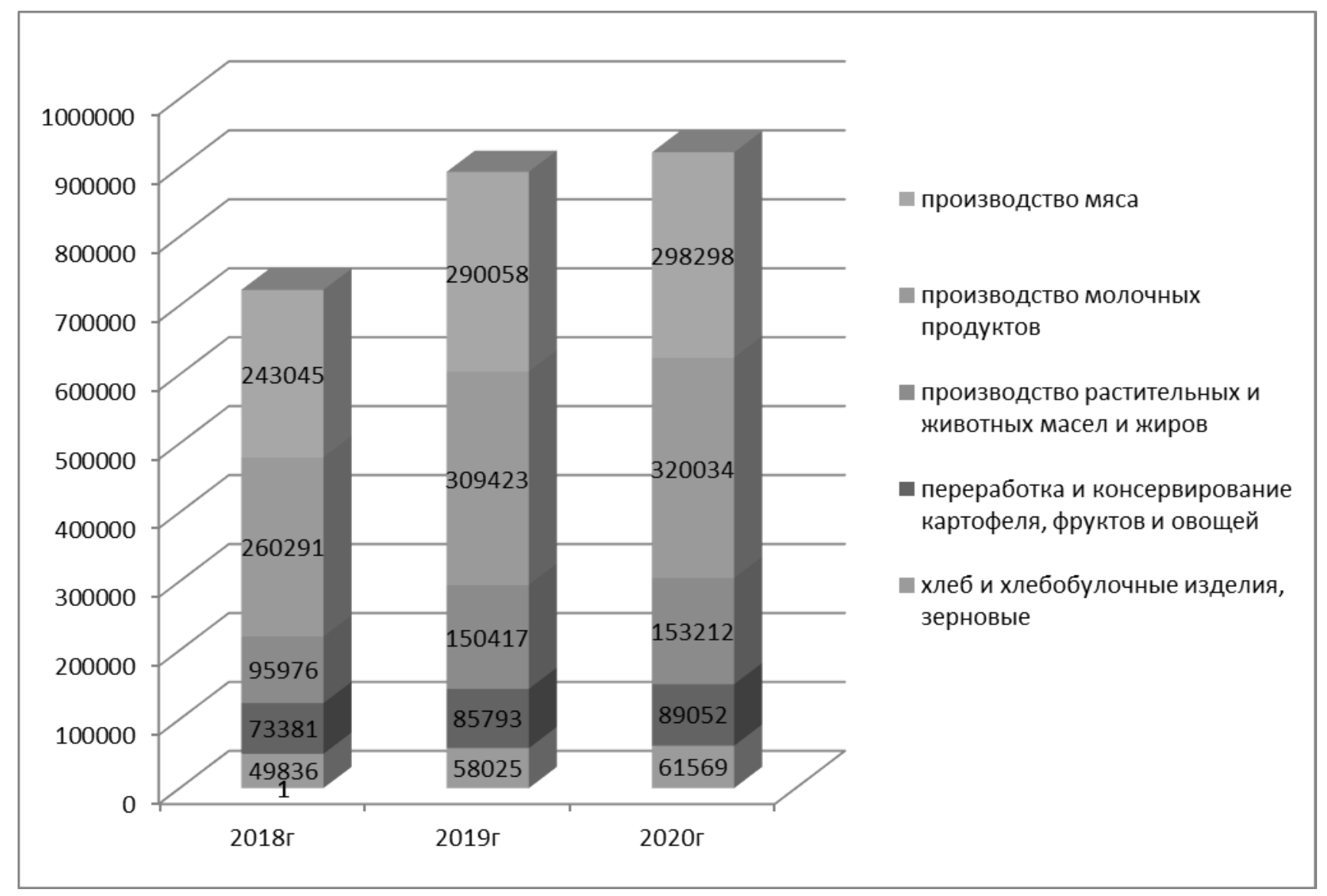

Рис. 4. Динамика производства промышленности и пищевой в сравнении по видам продукции, млн. руб. Источник: составлено автором по [2] 


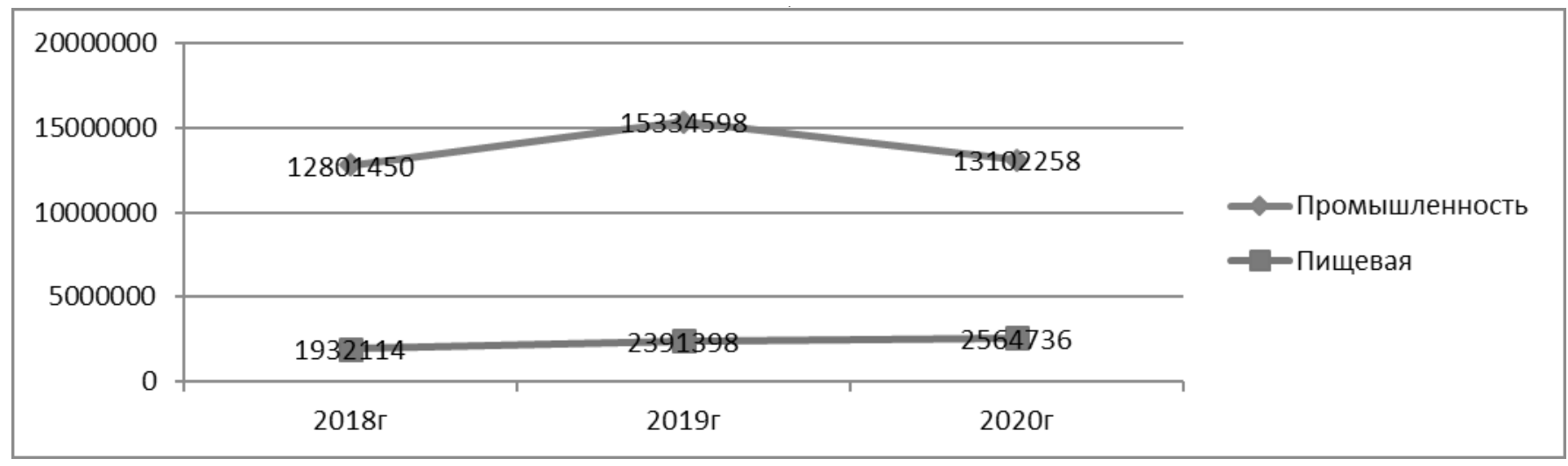

Рис. 5. Соотношение темпов роста производства в промышленности и пищевой, млн. руб. Источник: составлено автором по [2]

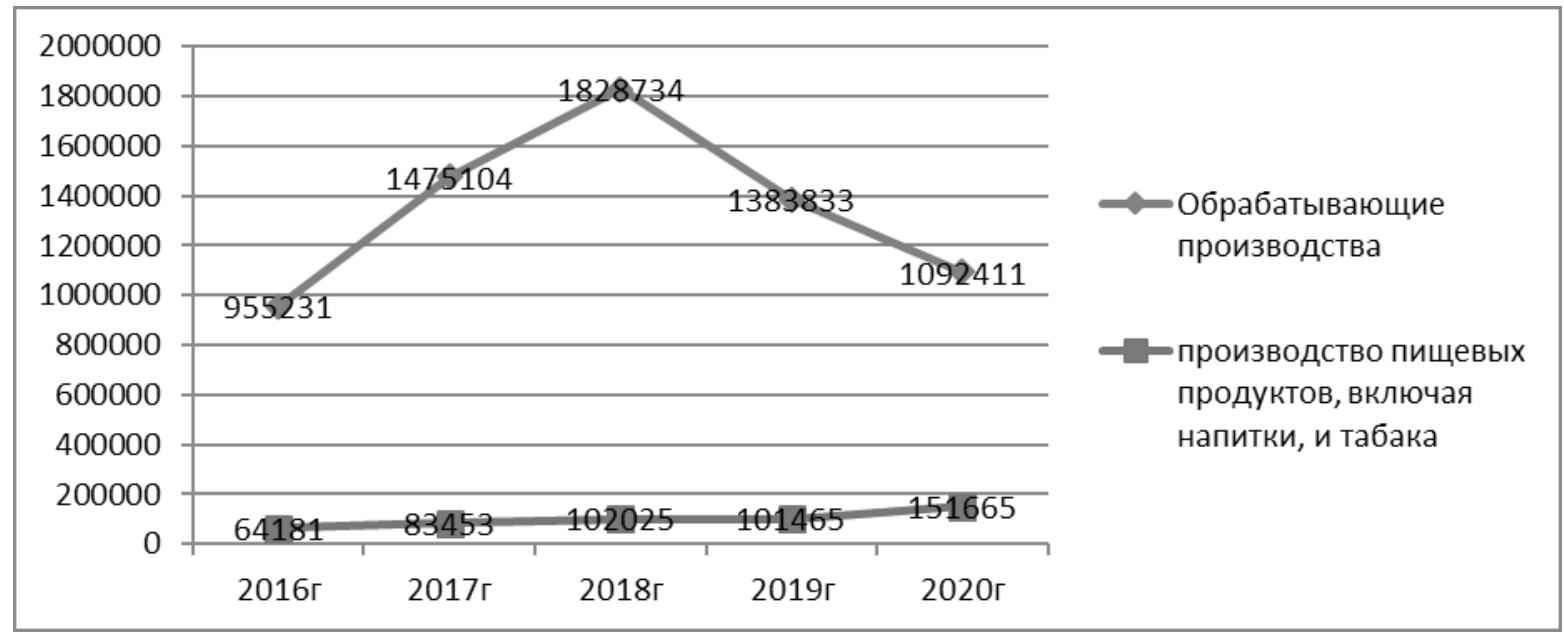

Рис. 6. Соотношение темпов роста финансовых результатов в промышленности и пищевой, млн. руб. Источник: составлено автором по [2]

нулась промышленности в целом, в пищевой промышленности данные явления были не так значительны.

Далее проведем сравнительный анализ числа предприятий в целом по промышленности и пищевой в частности (рисунок 3).

По сравнению с 2016 годом количество предприятий в промышленности снизилось, как в промышленности в целом, так и пищевой. Однако это не говорит об ухудшении работы отраслей. Данные тенденции связаны с повышением активности интеграционных движений в сторону объединения малых компаний в крупные холдинги, поскольку малому бизнесу все сложнее становится выстоять в сложных рыночных условиях.

Важным показателем в статистическом анализе, позволяющем выявить тенденции отрасли более четко, является динамика объема производства в натуральном и стоимостном выражениях.

Далее проведем сравнение динамики производства промышленности и пищевой в сравнении по видам продукции (рисунок 4).

Из рисунка 4 видно, что, несмотря на кризис, в 2020 году наблюдался прирост общего объема производства пищевой продукции. Это связано с ростом цен на пищевые товары, который наблюдался в 2020 году, а также спроса на них в виду кризисных явлений в экономике. Большая часть продаж приходится на производство молочной продукции и мяса. Высока доля производства растительных жиров и масел.

Далее следует провести анализ соотношения темпов роста объема производства в промышленности 


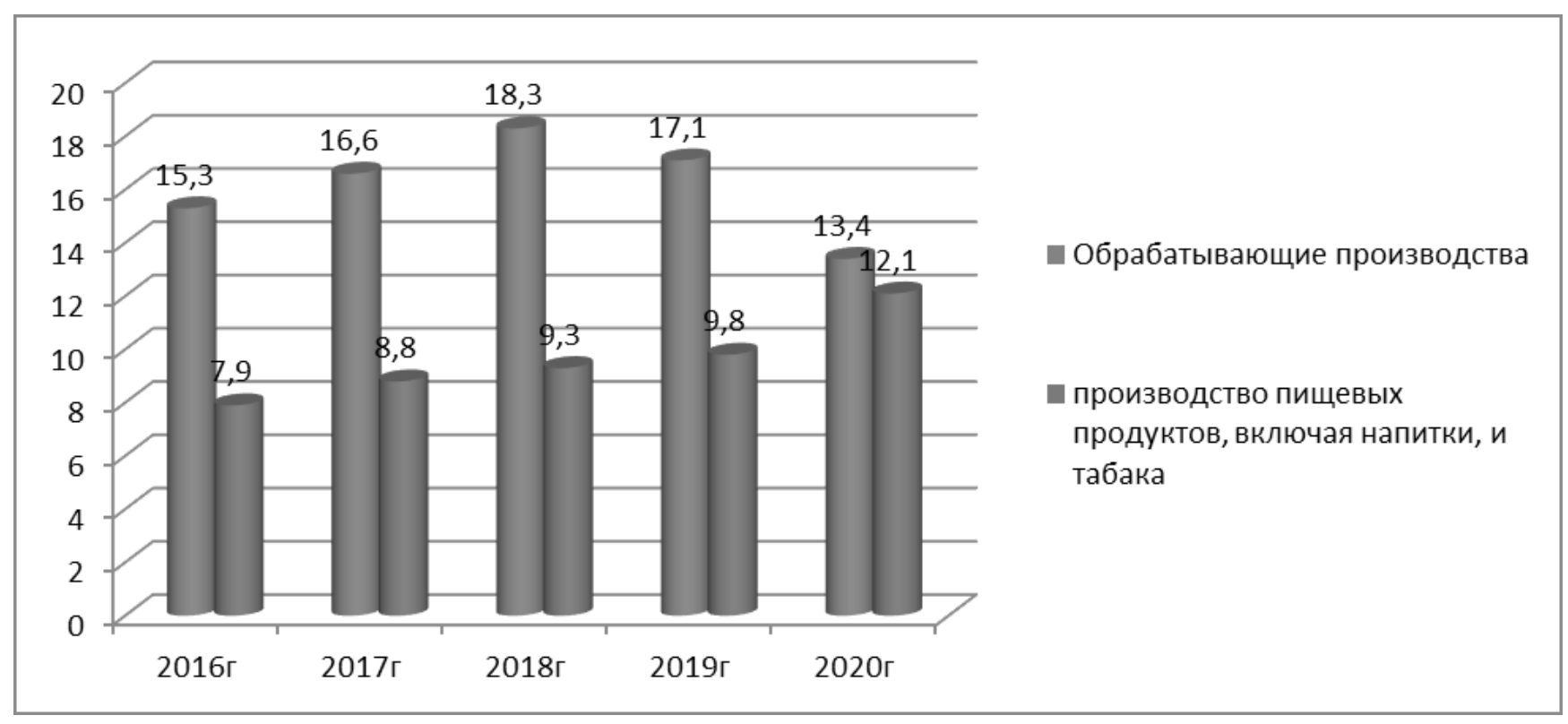

Рис. 7. Динамика показателей эффективности предприятий промышленности и пищевой,\% Источник: составлено автором по [2]

в целом и в пищевой. Данные для анализа приведены на рисунке 5 .

Данный график доказывает сделанные выше вывод, что не смотря на кризис в экономике в 2020 году пищевая отрасль развивалась достаточно стабильно. Имел место прирост объема производства, несмотря то, что в промышленности имелся значительный спад.

В заключении логично представить динамику финансовых результатов, полученных в промышленности в целом и пищевой отрасли в частности, которая приведена на рисунке 6.

Можно сделать выводы о том, что, несмотря на общий спад прибыли в связи с кризисными явлениями в экономике наблюдается некоторое увеличение финансовых результатов в пищевой промышленности. Для подтверждения указанных данных стоит представить анализ показателей рентабельности деятельности предприятий.

Основным показателем эффективности предприятия является рентабельность, анализ которой приведен на рисунке 7.

Рис. 7. доказывает, что при снижении среднего уровня рентабельности в промышленности в целом, в пищевой напротив наблюдается некоторый прирост в 2020 году, что и связано с увеличением объемов продаж и ростом цен в отчётном году в сравнении с базисным в целом по пищевой промышленности России.
Использование математических моделей в статистике позволяет выявить более четкие тренды и определить степень влияния на отрасль тех или иных факторов [3]. Проведем факторный анализ изменения объема производства продукции в 2020 году по сравнению с 2019 годом.

Объем производства продукции составил:

2019 г. у = 2398 млрд. руб.

2020 г. у $=2564$ млрд. руб.

Таким образом, объем производства продукции в 2020году увеличился по сравнению с 2019годом на166млрд. руб. (2564-2398) или на 21,3\% (2564/2398 = 1,069).

Определим влияние факторов на общее изменение объема производства продукции предприятиями пищевой отрасли промышленности. Факторная модель имеет вид [4]:

$$
\mathrm{y}=\mathrm{N}^{*} \mathrm{~d}^{*} \mathrm{~T},
$$

Где N - общая численность промышленно-производственного персонала;

D - удельный вес рабочих в общей численности ППП;

Т - производительность труда рабочих.

Вычислим производительность труда в 2019 и 2020 гг. 
Таблица 1.Сглаживание ряда динамики по методу 3-х летней скользящей средней

\begin{tabular}{|l|l|l|l|}
\hline Год & $\begin{array}{l}\text { Объем производства в ценах } \\
\mathbf{2 0 1 7} \text { г., млрд. руб. }\end{array}$ & $\begin{array}{l}\text { Скользящие суммы } \\
\mathbf{1} 1+\mathbf{y} 2+\mathbf{y} 3\end{array}$ & $\begin{array}{l}\text { Скользящие средние } \\
\text { (у1 +у2 +у3): 3, млрд. руб. }\end{array}$ \\
\hline 2016 & 1674 & - & - \\
\hline 2017 & 1885 & & 1830,3 \\
\hline 2018 & 1932 & 5491 & 2069,6 \\
\hline 2019 & 2392 & 6209 & 2296,3 \\
\hline 2020 & 2565 & $6889-$ & \\
\hline
\end{tabular}

Источник: составлено автором по [2]

Таблица 2. Расчет параметров уравнения прямой

\begin{tabular}{|c|c|c|c|c|c|c|}
\hline Год & $\begin{array}{l}\text { Условное } \\
\text { обозначение года, } \\
\mathrm{t}\end{array}$ & $\begin{array}{l}\text { Объем производства } \\
\text { продукции, млрд. } \\
\text { руб. у }\end{array}$ & $\mathbf{t}^{2}$ & $t^{*} y$ & $Y p$ & $(y-y p)$ \\
\hline 2016 & -2 & 1674 & 4 & 4 & -3348 & 5022 \\
\hline 2017 & -1 & 1885 & 1 & 1 & -1885 & 3770 \\
\hline 2018 & 0 & 1932 & 0 & 0 & 0 & 1932 \\
\hline 2019 & 1 & 2392 & 1 & 1 & 2392 & 0 \\
\hline 2020 & 2 & 2565 & 4 & 4 & 5130 & -2565 \\
\hline$\Sigma$ & 0 & & 10 & & & \\
\hline
\end{tabular}

Источник: составлено автором по [2]

2019 г.Т0 = 2398: 68,5 = 35 млн.руб. на чел.

2020 г.Т1 = 2564: 67,3 = 38,1 млн.руб. на чел

1. За счет увеличения численности промышленно-производственного персонала объем производства продукции увеличился на 33,9 млрд. руб.

$\Delta \mathrm{y}(\mathrm{N})=\Delta \mathrm{N} * \mathrm{~d} 0 * \mathrm{T0}=(68,5-67,3)^{*} 0,807 * 35=33,9$ млрд. руб.

2. За счет снижения доли рабочих в общей численности ППП объем производства продукции сократился на 32,9 млрд. руб.

$\Delta \mathrm{Y}(\mathrm{d})=\mathrm{N} 1 * \Delta \mathrm{d} * \mathrm{T0}=67,3^{*}(0,793-0,807) * 35=-32,9$ млрд. руб.

3. За счет повышения производительности труда объем производства продукции увеличился на 183106,0 млн. руб.

$\Delta \mathrm{Y}(\mathrm{T})=\mathrm{N} 1{ }^{*} \mathrm{~d} 1{ }^{*} \Delta \mathrm{T}=67,3^{*} 0,793^{*}(38,1-35)=165,4$ млрд. руб.

$\Delta \mathrm{Y}=\Delta \mathrm{Y}(\mathrm{N})+\Delta \mathrm{Y}(\mathrm{d})+\Delta \mathrm{Y}(\mathrm{T})=33,9-32,9+165,4=166,4$ млрд. руб.
Таким образом, наибольший удельный вес в увеличении выручки от реализации принадлежит влиянию повышения производительности труда.

Итак, посредством использования математической модели было выявлено, что основным фактором, повлиявшим на объем производства в отрасли, стал рост производительности.

Для того, чтобы составить прогноз развития отрасли, проведем сглаживание рассматриваемого ряда динамики по методу 3-х летней скользящей средней [5]. Результаты расчетов представим в таблице 1.

По данным таблицы 1 можно сделать вывод о тенденции увеличения уровня производства продукции пищевой отрасли с 2018 года.

Более совершенным приемом выявления общей тенденции в рядах динамики, на наш взгляд, является аналитическое выравнивание [6]. При проведении анализа общей тенденции путем аналитического выравнивания стоит учитывать уровни рядов динамики, которые могут быть с определенной степенью приближения к определенными математическими функциями. Таким образом, на основе теоретического анализа 
имеется возможность выявления характера развития отрасли во времени.

Далее проведем выравнивание данного ряда динамики по уравнению прямой:

$$
y t=a 0+a 1^{*} t
$$

Где а0, a1 - параметры уравнения прямой;

$\mathrm{t}$ - условное обозначение года.

Параметры уравнения прямой найдем методом наименьших квадратов, используя систему линейных уравнений.

$$
\begin{aligned}
& \mathrm{a} 0 * \mathrm{n}+\mathrm{a} 1^{*} \Sigma \mathrm{t}=\Sigma \mathrm{y} \\
& \mathrm{a} 0 \Sigma \mathrm{t}+\mathrm{a} 1 \Sigma \mathrm{t}^{2}=\Sigma \mathrm{ty}
\end{aligned}
$$

Расчет параметров уравнения прямой проведем в таблице 2.

Уравнение прямой имеет вид Уt $=520,96+72,91 \mathrm{t}$

По уравнению прямой можно сделать следующий вывод, что с каждым последующим годом объем производства продукции предприятиями пищевой отрасли будет повышаться.

Итак, предоставленный пример анализа основных показателей развития пищевой промышленности позволяет сделать вывод о значимости математических моделей в проведении данного анализа. При этом имеется возможность вычисления прогнозных значений, а также выявления факторов, ставших ключевыми в тех или иных тенденциях.

\section{ЛИТЕРАТУРА}

1. Долгова В.Н., Медведева Т.Ю. Теория статистики. Учебник и практикум для академического бакалавриата. М.: Юрайт, 2019. 246 с.

2. Деловой климат в промышленности в 2020 г.- М.: НИУ ВШЭ, 2020. - 24 с.

3. Малых Н.И. Статистика в 2-х томах. Том 2. Социально-экономическая статистика. Учебник и практикум для академического бакалавриата. М.: Юрайт, 2017. 474 c.

4. Статистика с элементами эконометрики. Учебник для СПО. В 2-х частях. Часть 2 / ред. Ковалев В.В. М.: Юрайт, 2019. 348 с.

5. Трофимов А.Г. Математическая статистика. Учебное пособие для СПО. М.: Юрайт, 2019. 260 с.

○ Кандалова Марина Андреевна ( renemaas@yandex.ru ), Бею Вероника Владимировна.

Журнал «Современная наука: актуальные проблемы теории и практики» 